does not generally recur after the first development, having produced in the constitution a certain inexplicable change. In lues, on the contrary, a person may be affected with constitutional symptoms time after time. These examples are not of frequent occurrence, but the fact is certain.

Persons who have suffered from secondary symptoms are generally more cautious for a time, and if they do contract a fresh infection, they seldom delay long in applying for advice, and hence constitutional symptoms are not very often observed in the same individual a second time.

The syphilitic poison does not observe a definite course, but runs on, and would, if unchecked, destroy its victim. There are no natural powers which can so alter its great activity as to render the poison innoxious. When the poison attacks the skin or throat, a process goes on, similar to that of an abscess, and the matter may be again absorbed to aggravate the disturbance of the system.

When no mercury has been used, the symptoms, after about a fortnight, will be less severe, as if the activity of the poison were suspended; and if too small a quantity have been taken, the same symptoms will appear and disappear several times.

Amongst the first class of secondary symptoms are to be reckoned ulcers on the tonsils, urula, and velum palati; blotches on the skin; lippitudo; ulcers on the umbilicus, on the nipple, under or between the toes, and on the penna nasi and upper lip; sarcocele, tinnitus aurium, intertrigo, rhagades, \&c.

(To be continued.)

\section{ON RETROFLEXION OF THE UTERUS.}

A Paper read before the Medical Society of King's College, London, Nov. 18, 1847.*

By Frederick John Hensley, M.B., Lond., VicePresident of the Society.

is

The canses which lead to this displacement of the womb may be divided into predisposing and exciting.

The predisposing are,-that congenital formation of the uterus, mentioned by Tiedemann, in which the uterus is situated obliquely, and the ligaments are lengthened; slight inclination and great capacity of the pelvis; relaxation of the tissues in the pelvis in general, and particularly of the broad and round ligaments; frequent abortions, by which the body of the uterus is rendered larger and heavier, and its neck relaxed and elongated, from repeated floodings; any canse which produces fulness and enlargement of the fundus, as fibrous tumours in that portion of the organ.

The exciting causes are,-accumulation in the bowels, whereby the pressure of the loaded ileum gradially forces down the fundus; falls; violent efforts to lift

- Continued from page $14_{\text {, }}$ heavy weights, or any other inordinate exertion. by which the abdominal muscles are brought strongly into. action; straining at stool, and these causes of course act with greater force when they concur with any of the predisposing causes. Dr. Denman considered retention of urine to be the cause in the single case related to him by Dr. Thomas Cooper; it is, probably, more an effect than a cause. Tumours in neighbouring organs, as the ovaries, may produce the same effect.

Let us now proceed to consider the effects produced by this displacement, and which add greatly to the importance of an early diagnosis. They are numerous and serious. Time will not allow me to enter minutely into their symptoms, which indeed would form an ample subject for another communication, I shall, therefore, content myself with a brief enumeration of them.

In all cases where the fundus has remained displaced. for some little time, congestion and engorgenent, not only of itself, but of the cervix and os uteri, is produced, owing to the return of venous blood being retarded; this congestion is occasionally so great as to lead even experienced men into the belief that malignant disease of the os and cervix is present, or that the retroflected fundus is a fibrous tumour.

The cause being overlooked, and the congestion continuing, ulcerative infammation of the cervix is apt to be set up, and a long train of severe symptoms ensues, such as pain and heat in micturition, bearing down, leucorrhœe, the discharge being yellow and thick, occasionally tinged with blood, and other symptoms too numerous to enter into. The relations of an organ whose sympathies extend through the whole female frame, insomuch that some writers have considered it the centre of female nervous sympathies, cannot be disturbed without generally producing many sympathetic affections, among which we may class. nausea, romiting, spasms, and the protean forms of hysteria.

Another affection very commonly associated with retroflexion, and to which $I$ have alluded in describing the symptoms, is oophoritis, the peculiar symptoms of which are pain, heat, and tenderness in the corresponding groin, a sense of weight and swelling there, (the latter may be sometimes distinguished through the abdominal walls,) and what may, united with these symptoms, be almost regarded as pathognomionic of this affection, lameness of one leg, the patient being unable to extend the thigh without pain; she keeps it flexed on the abdomen. On examination per vaginam. the swelled ovary may be felt, it is, however, more easily detected per rectum as an oval swelling posterior to and above the uterus, very tender to the touch.

Other effects are sterility, tendency to abortion, and. dyamenorrbnea.

Retroflexion of the fundus tends, I believe, to produce 
abnormal growth in the substance of the uterus, especially fibrous tumours, by occasioning congestion, and keeping up irritation and an increased flow of blood to the part. This is a point requiring further investigation.

Having thus described generally the symptoms, history, causes, and effects, of this very important displacement, I will proceed to illustrate the disease by the detail of one or two cases, premising that it is not to be expected that all the symptoms $I$ have enumerated should be present in any individual case, and that the treatment adopted will be described more fully afterwards. For the first case I am indebted to Dr. Rigby's reports.

L. B-, aged 27, married three years, does not suppose kerself ever to have been pregnant, tall, and well formed.

August 4th, 1845. Catamenia have always been regular, lasting six or seven days, but since her marriage they have been rather more profuse, lasting eight, or even ten, days; slight leucorrhca during the intervals; digestion usually good; bowels confined; accustomed to active exercise; pulse very feeble; has a remarkably dry skin, which has been peculiar to her since her childhood; the feet have an irritable eruption on them, which at times itches severely. When she perspires, which rarely happens, it seems to relieve this source of irritation considerably; urine always high coloured, and turbid on cooling, occasionally with red sand, but never scanty.

Examination per vaginam :-Os uteri looking almost directly forward; the fundus uteri can be felt behind the vagina; uterine sound passed as far as the os uteri internum, the point being directed backwards, and by raising the fundus a little with the examining finger, the sound passed into the uterine cavity to its full extent. I then raised the fundus upwards and forwards into its natural position. She states that a very slight appearance of the catamenia occurred shortly after marriage; that in about a fortnight afterwards she received a severe concussion of the brain, in consequence of being thrown from a carriage with great violence, when it was upset. She went ten days beyond the next period; a slight show then came on, and again disappeared, but in twenty-four hours it returned very profusely, with an unusual degree of pain, and with the expulsion of several large clots.

R. Extr. Taraxaci, cochl. min. $\frac{1}{2}$, omni nocte e lacte. R. Pulv. Guaiaci, Magn. Carb., utrq, gr. x., ex Aqua, cum Sp. Ammon. Aromat., dr. ss., omni meridie.

She left town immediately afterwards.

October 3rd. She returned to London on Sept. 30th, in good health; the course of medicine which she has been taking seems to have agreed well, and to have diminished the lithic diathesis, which formerly existed. I introduced an ivory supporter, fixed to a pessary as before described; it appeared to answer the purpose completely without producing any inconvenience; I therefore omitted to use an astringent lotion, as I expected that the contraction of the vagina would be sufficient to retain it in position. On her rising, however, into the upright posture, it slipped and cathe down the same evening.

4th. Introduced an irory pin of the same flattened form as yesterday, and fixed it to Dr. Simpson's instrument for supporting the uterus; it was adapted with much ease, the os uteri having been gently dilated previously to the uterus being raised into its natural position.

7th. Rectified the instrument by slightly bending it, as it pressed more on one labium than the other.

10th. Catamenia appeared without pain; bealth good.

16th. Leaves town to-morrow to visit her parents at a considerable distance.

December 10th. I visited her for the purpose of removing the supporter, but on my arrival $I$ found that two days previously, when rising from her chair, she accidentally trod on one of the tapes and pulled it entirely out.

Examination per vaginam.-The os uteri opened backwards and rather low down, the uterine sound passed easily in the right direction.

Dr. Rigby remarks, " The above case presents several features of interest. In the first place a considerable degree of retroflexion is ascertained to exist without its producing any derangement or inconvenience whatever; the only change which could have been attributed to it was the circumstance of the catamenia having been rather more profuse since than before her marriage. No trace of dysmenorrhœa or ovarian irritation had existed, not even the slightest degree of pain or uneasiness about the pelris, nor was she at all aware of any difference in her feelings, either after $I$ had replaced it, or when it returned to its former retroflected condition."

The history of the patient from the time of her marriage, to the unusually profuse catamenial period, which followed her accident, is interesting:-" $A$ very slight appearance of the catamenia occurred shortly after marriage," a circumstance, where this function had been so regular, which would favour the supposition that conception had taken place, the more so as she was in perfect health at the tirne; then came the accident, which was accompanied by so serere a concussion, as to render ber more or less insensible even for some days. She went ten days beyond the ordinary period, which is exactly the reverse of her usual habit, being inclined to have a return of the catamenia some few days short of the full time. The manner in which the discharge appeared was equally unusual :- " a slight show came on, again disappeared; but in twenty-four hours it returned very profusely, with an unusual degree of pain, and with the expulsion of several largs clots." The whole features of this period seem to me to indicate the occurrence of an extremely early abortion.

The necessary inference appears to be that conception had taken place very shortly after marriage; that she 
was exposed to a serious concussion of the body as well as of the head, when adranced nearly three weeks in pregnancy, and when the uterus therefore was beginming to undergo that increase of vascular congestion, which is known to be one of the earliest changes it presents after conception, and by which its fundus being rendered more beavy and bulky than ordinary, would be peculiariy liable to be forced downwards and backwards by the occurrence of so serious an accident as that which she met with.

The next case I shall allude to is one which came under my own notice, and in which the symptoms were more serions. It is as follows :-

December, 1843. Marianne Nichol,* widow, aged 35 , has been married sixteen years; had six children, (the last three years ago,) and one miscarriage. Catamenia appeared at the age of eleven years, and the discharge was profuce for a time, but shortly stopped for six months, and again returned, and from this time she had a continued discharge for three years. The monthly discharge is always regular during pregnancy. Ten years ago had a very difficult labour, and from that time has always been ailing. About four years ago she overstrained berself by lifting a heavy box, and since then she has suffered from pain in the back, and other symptoms referrible to the pelvic organs. For the last four months has had a pale offensive discharge, and pain and heat in micturition. At present complains of pain in the loins and left groin; also of pain down the left leg, with numbness; the urine is scanty, and causes pain in passing it; there is no frequent desire to pass it; bowels costive; has been in the habit of taking aperient medicines; defæcation gives great pain. The catamenia are preceded by much pain of a bearing-down character, shooting down the thighs, especially the left; the discharge is copious, dark-coloured, and attended with fibrous exudations and pain in the back; slimy leucorrhœa occurs in the intervals.

Examination per vaginam :-The os uteri is tender to the touch, and bleeds readily on pressure.

Examination per speculum:-The anterior lip is divided into several lobules, it is large and swollen; the posterior lip is covered with highly vascular granulations, and is fissured; the os uteri looks downwards, not backwards; behind the posterior lip is a tumour, hard and painful.

The nitrate of silver was applied to the uterus; Lotio Plumbi ordered for injection; quinine and sulphuric acid taken internally.

5th. Catamenia have returned this morning; feels very low and weak; suffers a good deal from dyspnœea.

On the 9th was ordered calomel and colocynth pill, and Acidum Hydrocyanicum, with Inf. Quassiæ, for the dyspeptic symptoms.

It would be tedious and irrelevant to the subject to enter into the symptoms dependent on dyspepsia, and

- Admitted into King's College Hospital, under Dr. Todd, December, 1845 an affection of the chest; I suall therefore confine myself to the uterine symptoms.

18th. The leucorrhoea has continued copions and offensive. To-day I scarified the os uteri, but it afforded little relief, as it did not bleed freely. The next day I applied eight leeches, which bled freely.

29th. Feels better; the catamenia have appeared, and are attended with less pain and bearing-down. On first trial I was unable to pass the sound, but on the 9th of January, having curved it more, I succeeded in doing 80 ; it was felt in the tumour behind the vagina, and by turning it upwards and forwards, I remored the tumour, and restored the fundus to its position. The body was bent backwards, and to the right side; its continuity with the os was traceable by the finger.

She was discharged on the $30 \mathrm{th}$, as I had obtained an order for her admission into the hospital for women, into which hospital she was admitted on the 10th of February, under Dr. Protheroe Smith. The retroflexiou was found to have returned again, and the tumour was tender and painful.

Hirudines vj. cervici uteri applicandæ.

February 25th. The uterus was restored to its natural position by the aid of the uterine sound.

Hirudines vj. ori uteri.

28th. After the uterus had been restored on the $25 \mathrm{th}$, there was great pain in the back and loins; leeches bled freely; bowels open thoroughly on the 25th.

Examination per vaginam:-The anterior lip is hard and nodulated; the os looks backwards into the hollow of the sacrum; the uterine sound passes easily to its full extent, with its concavity forwards, while there is now no tumour behind the neck of the womb.

March 3rd. The uterns is again distinctly retroflexed, as evidenced by the introduction of the uterine sound; bowels open. Hirudines vj.

20th. Uterus in its natural position; uterine sound passes with its concavity forward, and no tumour can be felt posteriorly.

27 th. Uterus in its natural position. The patient is very hysterical.

3Ist. Uterine sound passes to three inches and a half in its natural direction, with its concavity forwards; the cervix is large and bulky; os irregular and indurated; no tumour posteriorly; the posterior pouch of the vagina indurated.

Pil. Ferri Sulph., gr. v., ter die sumend.

April 14th. Feels much better, but still complains of pain at the chest; a sensation as of hot water in the stomach; has a very offensive discharge; the pain in left groin has entirely disappeared, leaving a slight tenderness.

Examination per vaginam:-Uterus in proper position; the os less indurated; uterine sound passes with concavity forwards, and half an inch beyond the natural extent; the os looks distinctly backwards; and there is no pelvic tumour posteriorly.

She was subsequently made an out-patient. Occasional scarifications and mercurial pastiles were ordered, which after a time affected the system; a progressire improvement in the condition of the cervix. 
and os took place, though the retroflexion returned once and again, but she was ultimately discharged cured.

This case furnishes an instance of the great tendency which exists in cases of long standing to a return of the retroflexion; it was remarkable for its obstinacy. The long duration of the treatment necessary to produce cure should not excite our wonder, when we consider that the disease had evidently existed for some years, and had been overlooked, and consequently neglected ; that it had led on to excessive engorgement of the cervix and fandus, and ulceration of the posterior lip; that the body and cervix were elongated, so that the sound at one time passed three inches and a half, and therefore Simpson's supporter wasinapplicable. It was only after the ulceration had been bealed by cauterizations, and the voluminous organ reduced by repeated leechings, and local mercurialization, that the disease was cured.

I might bring before you numerous other cases which have come under my own notice, but having detailed these, I deem them sufficient for my present purpose, and will not detain you long by dwelling on the general treatment.

In the first place the causes should be removed; the bowels must be unloaded, and kept gently open by saline aperients; the general health improved by alteratives and tonics; local engorgement relieved by leeches applied to the anus, or to the os and cervix uteri, followed by the warm hip-bath. The fundus must then be replaced by the uterine sound, and the patient enjoined to remain in bed on the side for some days. If the fundus remains in its proper position, so much the better; astringent injections will be all that is required. If otherwise, the uterus must be again restored, and perhaps by keeping the sound a short time in it, we may overcome the tendency.

Should this means fail, we must employ the uterine supporter devised by Professor Simpson, an instrument which I now exhibit to you. It consists of a metallic or irory pin, the length of the uterine cavity, (two inches and a balf,) fixed in a disc or button, on which the os uteri rests, connected with and kept in position by a little frame, resting on the mons veneris, and which is properly fastened by tapes. Dr. Rigby improved this instrument by making the pin flat instead of round, and broader at the extremity, so as to adapt it to a larger surface, and by employing ivory instead of metal. Dr. Simpson had remored the objection of corrosion being produced by the secretions, by having the metal electro-gilded. The instrument should be adapted while the patient is in bed, and she should be kept quiet for some days, till the uterus becomes accustomed to its presence. I have known peritonitis induced by the neglect of this precaution, the patient having walked home some distance; where, however, the patient is cautious in her movements, and disposed to follow the directions of her medical attendant, the instrument may be worn for monthe without inconvenience, and excites less irritation or discharge than the ordinary pessaries. The instrument is generally required to be worn a month or six weeks to effect a cure; after wearing it a short time the patient is able to take moderate exercise.

Dr. Simpson has another instrument for the same purpose, it is a species of pessary, to which he has fired a hinge, by means of a spring, like the blade of a knife, but this I have neither used nor seen.

Into the treatment of the complications, it is impossible to enter minutely, I fear I have already trespassed too long on your time and patience.

\section{SYMPTOMS OF POISONING, AFTER EATING A QUANTITY OF MACE.}

By Grorge C. Watson, M.D., Liverpool, formerly Lecturer on Forensic Medicine, in Dablin.

(Read before the Liverpool Medical Society. 1847.)

The subjoined toxicological effects of an article extensively used as a condiment, will, perhaps, justify their being placed on record under the head of narcoticoacrid, or narcotico-irritant ; and it is the more necessary to establish the concentrated physiological action of the nutmeg and its singular envelope, because its culinary merits under a diluted form render its milder effects a subject of common experience, while its more intense action appears to be little known, at least as far as $\mathbf{m y}$ research has carried me.

The entire indigestibility of the compact fibre of the arillus of the nutmeg is, perhaps, only matched by the nut it encloses, and suggests at once as the first step of treatment, an emetic, in order to remove from the stomach a substance which, many hours even after having been taken, shews great resistance to the combined processes of mastication and digestion, and requires a lengthened period for the full development of its toxicological effects. Most probably to this cause is to be referred the slow accession of the symptoms in the first place, and their obstinately prolonged duration.

Perhaps it might be doubted whether the treatment subsequent to the emetic was the most suitable, especially the selection of a remedy like camphor, which belongs to the same class of poisons as the one to which $I$ have rentured to refer the present drug; but the small. ness of the doses of camphor given puts it almost "out of court." The other diffusible stimuli seemed indicated by the symptoms, \&c.

"At about eleren o'clock in the forenoon of the 12th, of March, 1847, I was in a sale-room in which there were samples of spices, \&c.; and from curiosity, I ate about as much mace as coald be lifted on a teaspoon, if broken small. About half-past twelfe o'clock I felt 